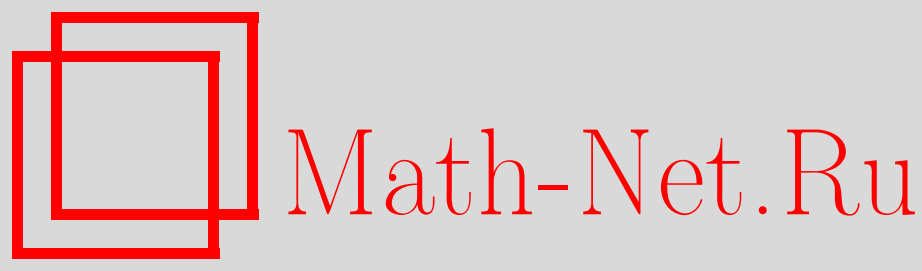

Л. Ц. Аджемян, С. Е. Воробьева, Э.В.Иванова, М. В. Компаниец, Представление ренормгрупповых функций несингулярными интегралами в модели критической динамики ферромагнетиков: четвертый порядок $\varepsilon$-разложения, $Т M \Phi, 2018$, том 195, номер 1, 105-116

DOI: https://doi.org/10.4213/tmf9412

Использование Общероссийского математического портала Math-Net.Ru подразумевает, что вы прочитали и согласны с пользовательским соглашением http: //www . mathnet.ru/rus/agreement

Параметры загрузки:

IP : 54.197 .130 .99

26 апреля 2023 г., 04:09:13

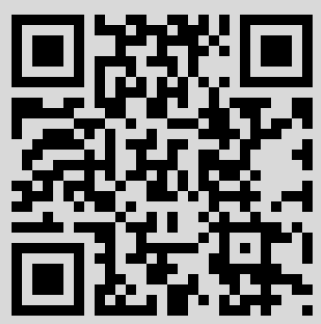




\title{
ПРЕДСТАВЛЕНИЕ РЕНОРМГРУППОВЫХ ФУНКЦИЙ НЕСИНГУЛЯРНЫМИ ИНТЕГРАЛАМИ В МОДЕЛИ КРИТИЧЕСКОЙ ДИНАМИКИ ФЕРРОМАГНЕТИКОВ: ЧЕТВЕРТЫЙ ПОРЯДОК
}

\begin{abstract}
С использованием представления для ренормгрупповых функций через несингулярные интегралы произведен расчет динамического критического индекса в модели критической динамики ферромагнетиков в четвертом порядке $\varepsilon$-разложения. Вычисления диаграмм Фейнмана проводились с помощью техники Sector Decomposition, обобщенной на задачи критической динамики.
\end{abstract}

Ключевые слова: ренормализационная группа, $\varepsilon$-разложение, многопетлевые диаграммы, критические показатели.

DOI: https://doi.org/10.4213/tmf9412

\section{1. ВВЕДЕНИЕ}

В работах [1], [2] предложен подход, позволяющий сводить вычисление $\beta$-функции и аномальных размерностей в моделях критической статики к расчету интегралов, не содержащих сингулярностей по $\varepsilon$, удобных для численного интегрирования. В статье [3] этот метод обобщен на задачи критической динамики. В настоящей работе данный подход использован для вычисления динамического критического индекса модели критической динамики ферромагнетиков (“модель А” в обзоре [4]) в четвертом порядке $\varepsilon$-разложения. Диаграммы Фейнмана были вычислены с использованием техники Sector Decomposition [5].

Статья построена следующим образом. В разделе 2 изложена схема ренормировки, в рамках которой с использованием $R$-операции ренормгрупповые (РГ-) функции представлены в виде разложений, свободных от ультрафиолетовых (УФ) расходимостей. В разделе 3 получено представление Фейнмана для диаграмм динамической теории, необходимое для их вычисления посредством техники Sector Decomposition. В разделе 4 получена формулировка $R$-операции для диаграмм, проинтегрированных по времени. В разделе 5 приведены результаты четырехпетлевого расчета. В приложении изложены некоторые детали расчета диаграмм.

* Санкт-петербургский государственный университет, Санкт-Петербург, Россия. E-mail: l.adzhemyan@spbu.ru 


\section{2. РЕНОРМИРОВКА МОДЕЛИ}

Неренормированное действие модели $A$ критической динамики в пространстве размерности $d=4-\varepsilon$ определяется набором $\phi_{0}$ двух полей $\phi_{0} \equiv\left\{\psi_{0}, \psi_{0}^{\prime}\right\}$ и имеет вид [6]

$$
\begin{aligned}
S_{0}\left(\phi_{0}\right) & =\lambda_{0} \psi_{0}^{\prime} \psi_{0}^{\prime}+\psi_{0}^{\prime}\left[-\partial_{t} \psi_{0}+\frac{\lambda_{0} \delta S_{0}^{\mathrm{st}}}{\delta \psi_{0}}\right]= \\
& =\lambda_{0} \psi_{0}^{\prime} \psi_{0}^{\prime}+\psi_{0}^{\prime}\left[\partial_{t} \psi_{0}+\lambda_{0}\left(\partial^{2} \psi_{0}-\tau_{0} \psi_{0}-\frac{1}{3 !} g_{0} \psi_{0}^{3}\right)\right],
\end{aligned}
$$

где неренормированное статическое действие

$$
S_{0}^{\text {st }}\left(\psi_{0}\right)=-\frac{\left(\partial \psi_{0}\right)^{2}}{2}-\frac{\tau_{0} \psi_{0}^{2}}{2}-\frac{1}{4 !} g_{0} \psi_{0}^{4} .
$$

Ренормированное действие $S_{\mathrm{R}}=S_{\mathrm{B}}+\Delta S$ представляет собой сумму базового действия $S_{\mathrm{B}}$ и контрчленов $\Delta S$, с исходным действием (1) оно связано мультипликативной перенормировкой параметров [6]:

$$
\begin{aligned}
& S_{\mathrm{B}}=\lambda \psi^{\prime} \psi^{\prime}+\psi^{\prime}\left[-\partial_{t} \psi+\lambda\left(\partial^{2} \psi-\tau \psi-\frac{1}{3 !} \mu^{\varepsilon} g \psi^{3}\right)\right] \\
& S_{\mathrm{R}}=Z_{1} \lambda \psi^{\prime} \psi^{\prime}+\psi^{\prime}\left[-Z_{2} \partial_{t} \psi+\lambda\left(Z_{3} \partial^{2} \psi-Z_{4} \tau \psi-\frac{1}{3 !} Z_{5} \mu^{\varepsilon} g \psi^{3}\right)\right],
\end{aligned}
$$

где

$$
\begin{aligned}
& \lambda_{0}=\lambda Z_{\lambda}, \quad \tau_{0}=\tau Z_{\tau}, \quad g_{0}=g \mu^{\varepsilon} Z_{g}, \quad \psi_{0}=\psi Z_{\psi}, \quad \psi_{0}^{\prime}=\psi^{\prime} Z_{\psi^{\prime}}, \\
& Z_{1}=Z_{\lambda} Z_{\psi^{\prime}}^{2}, \quad Z_{2}=Z_{\psi^{\prime}} Z_{\psi}, \quad Z_{3}=Z_{\psi^{\prime}} Z_{\lambda} Z_{\psi} \\
& Z_{4}=Z_{\psi^{\prime}} Z_{\lambda} Z_{\tau} Z_{\psi}, \quad Z_{5}=Z_{\psi^{\prime}} Z_{\lambda} Z_{g} Z_{\psi}^{3} .
\end{aligned}
$$

Из мультипликативной ренормируемости моделей (1), (2) следует, что динамические константы ренормировки $Z_{\psi}, Z_{\tau}, Z_{g}$ совпадают со статическими и справедливо соотношение $Z_{\psi^{\prime}} Z_{\lambda}=Z_{\psi}[6]$. Это означает, что константы ренормировки $Z_{3}, Z_{4}, Z_{5}$ являются чисто статическими и

$$
Z_{1}=Z_{2}
$$

Единственной новой константой ренормировки является

$$
Z_{\lambda}=Z_{1}^{-1} Z_{\psi}^{2}=Z_{2}^{-1} Z_{\psi}^{2} .
$$

Как и в статье [2], выберем схему ренормировки, аналогичную схеме вычитаний на нулевых импульсах и частотах, с дополнительным условием $\mu^{2}=\tau$. Константы ренормировки определим из требований

$$
\left.\bar{\Gamma}_{i}^{\mathrm{R}}\right|_{\mu^{2}=\tau}=1, \quad i=1, \ldots, 5,
$$

где $\bar{\Gamma}_{i}^{\mathrm{R}}$ - ренормированные аналоги следующих функций (все на нулевых частотах $\omega=0$ и импульсах $p=0)$ :

$$
\begin{gathered}
\bar{\Gamma}_{1}=\frac{\Gamma_{(0,2)}}{2 \lambda}, \quad \bar{\Gamma}_{2}=\partial_{\imath \omega} \Gamma_{(1,1)}, \\
\bar{\Gamma}_{3}=-\frac{1}{2} \partial_{p}^{2} \frac{\Gamma_{(1,1)}}{\lambda}, \quad \bar{\Gamma}_{4}=-\frac{\Gamma_{(1,1)}-\left.\Gamma_{(1,1)}\right|_{\tau=0}}{\lambda \tau}, \quad \bar{\Gamma}_{5}=-\frac{\Gamma_{(3,1)}}{\lambda g \mu^{\varepsilon}},
\end{gathered}
$$


и введено обозначение для 1-неприводимых функции Грина базовой теории (3)

$$
\Gamma_{\left(n_{1}, n_{2}\right)} \equiv\langle\underbrace{\psi \cdots \psi}_{n_{1}} \underbrace{\psi^{\prime} \cdots \psi^{\prime}}_{n_{2}}\rangle_{1-\mathrm{irr}}
$$

В используемой схеме ренормировки константы ренормировки $Z_{i}$, как и в схеме минимальных вычитаний, зависят лишь от заряда $g$ и размерности пространства, а определяемые этими константами РГ-функции ( $\beta$-функция и аномальные размерности $\gamma_{i}$ ) даются выражениями

$$
\beta(g)=-\varepsilon \frac{g}{1+g \partial_{g} \ln Z_{g}}, \quad \gamma_{i}(g)=-\varepsilon \frac{g \partial_{g} \ln Z_{i}}{1+g \partial_{g} \ln Z_{g}} .
$$

Динамический критический индекс определяется единственной новой по сравнению со статикой РГ-функцией $\gamma_{\lambda}$, для которой мы будем использовать вытекающие из формул (6)-(8), (12) соотношения

$$
\gamma_{\lambda}=2 \gamma_{\psi}-\gamma_{1}, \quad 2 \gamma_{\psi}=\gamma_{3}
$$

Учет контрчленов можно заменить действием $R$-операции на диаграммы базовой теории (3) и записать ренормированные функции $\Gamma_{\left(n_{1}, n_{2}\right)}^{\mathrm{R}}$ в виде [6]

$$
\Gamma_{\left(n_{1}, n_{2}\right)}^{\mathrm{R}}=R \Gamma_{\left(n_{1}, n_{2}\right)}=(1-K) R^{\prime} \Gamma_{\left(n_{1}, n_{2}\right)},
$$

где операция $R^{\prime}$ устраняет расходимости в существенных подграфах диаграммы, а $(1-K)$ - остающуюся поверхностную расходимость. Полную $R$-операцию можно записать в виде [7]

$$
R \Gamma=\prod_{i}\left(1-K_{i}\right) \Gamma
$$

где произведение берется по всем существенным (имеющим поверхностную расходимость) подграфам диаграмм, входящих в Г и диаграммам в целом (если у них есть поверхностная расходимость).

Теория ренормировок гарантирует УФ-конечность РГ-функций (12) - сокращение полюсов по $\varepsilon$ в правых частях этих соотношений, однако сингулярный характер констант ренормировки весьма затрудняет их определение численными методами. В работе [3] для расчета РГ-функций предложено использовать соотношения

$$
\gamma_{i}=\frac{2 f_{i}}{1+f_{3}}, \quad i=1,2,3,5
$$

где

$$
f_{i}=-\left.R \tau\left(\partial_{\tau} \bar{\Gamma}_{i}\right)\right|_{\mu^{2}=\tau}=\left.\tau R \bar{\Gamma}_{i}^{(1)}\right|_{\mu^{2}=\tau}
$$

Операция $-\partial_{\tau} \bar{\Gamma}_{i}$ соответствует сумме всевозможных вставок составного оператора $\Phi=\psi^{\prime} \psi$ в линии диаграмм функции $\bar{\Gamma}_{i}$, поэтому величина $\Gamma_{i}^{(1)}$ имеет смысл соответствующей 1-неприводимой функции со вставкой составного оператора $\Phi$. 1-Неприводимые функции $\bar{\Gamma}_{i}^{(1)}$ с $i=1,2,3,5$ не имеют поверхностной расходимости, поэтому для них $R$-операция в формуле (15) сводится к $R^{\prime}$-операции. Все величины $f_{i}$ не зависят от $\mu$ и $\tau$. 
При вычислении величин $f_{i}$ с использованием представления (15) операции вычитания $1-K_{i}$ в используемой схеме ренормировки сводятся к вычитанию начального отрезка ряда по импульсу и частоте. Для логарифмических подграфов имеем

$$
(1-K) \Gamma\left(p^{2}, \omega\right)=\Gamma\left(p^{2}, \omega\right)-\left.\Gamma\right|_{p=0, \omega=0},
$$

для квадратичных

$$
(1-K) \Gamma\left(p^{2}, \omega\right)=\Gamma\left(p^{2}, \omega\right)-\left.\Gamma\right|_{p=0, \omega=0}-\left.p^{2} \partial_{p^{2}} \Gamma\right|_{p=0, \omega=0}-\left.i \omega \partial_{i \omega} \Gamma\right|_{p=0, \omega=0} .
$$

В обоих случаях операцию вычитания можно записать в виде остаточного члена ряда Тейлора в интегральной форме:

$$
(1-K) \Gamma\left(p^{2}, \omega\right)=\int_{0}^{1} d a \partial_{a} \Gamma\left(a p^{2}, a \omega\right)
$$

для логарифмических подграфов и

$$
(1-K) \Gamma\left(p^{2}, \omega\right)=\int_{0}^{1} d a(1-a) \partial_{a}^{2} \Gamma\left(a p^{2}, a \omega\right)
$$

- для квадратичных. Действие операции $R$ на диаграмму $\chi$ с учетом выражений (20), (21) можно записать в виде [7]

$$
R \chi=\prod_{i} \int_{0}^{1} d a_{i}\left(1-a_{i}\right)^{n_{i}} \partial_{a_{i}}^{n_{i}+1} \chi(\{a\}),
$$

где произведение берется по всем существенным подграфам $\chi^{(i)}$ (включая диаграмму $\chi$ как целое), $a_{i}$ - параметр растяжения внутри $i$-го подграфа импульсов и частот, втекающих в этот подграф; $n_{i}=0$ для логарифмических подграфов и $n_{i}=1$ для квадратичных. Преимущество такой записи ренормированных величин заключается в том, что ответ представляется в виде интегралов, конечных при $\varepsilon=0$, причем в форме, в которой не происходит сокращения больших вкладов в подынтегральном выражении (“теория без расходимостей”). Отметим, что вставка оператора $\Phi$ в логарифмический подграф делает его несущественным, а вставка $\Phi$ в квадратичнорасходящийся подграф делает подграф логарифмическим.

\section{3. ПРЕДСТАВЛЕНИЕ ФЕЙНМАНА}

В статической модели (2) переход к представлению Фейнмана основан на том, что в импульсном представлении подынтегральные выражения для диаграмм представляют собой произведения пропагаторов вида $1 /\left(k^{2}+\tau\right)$, зависящих от квадратов импульсов интегрирования. Представление для подынтегральных выражений в терминах фейнмановских параметров можно получить в этих случаях непосредственно по виду диаграмм.

Пропагаторы динамической модели (3) в импульсно-временном представлении имеют вид

$$
\begin{aligned}
& \stackrel{t_{1} \quad t_{2}}{\longrightarrow}=\left\langle\psi\left(t_{1}\right) \psi\left(t_{2}\right)\right\rangle=\frac{1}{E_{k}} e^{-\lambda E_{k}\left|t_{1}-t_{2}\right|}, \\
& \stackrel{t_{1} \quad t^{t_{2}}}{\overbrace{}^{2}}=\left\langle\psi\left(t_{1}\right) \psi^{\prime}\left(t_{2}\right)\right\rangle=\theta\left(t_{1}-t_{2}\right) e^{-\lambda E_{k}\left(t_{1}-t_{2}\right)},
\end{aligned}
$$

где $E_{k} \equiv k^{2}+\tau$. 
Для расчета РГ-функций требуется вычислять диаграммы на нулевой внешней частоте. Интегрирование по промежуточным частотам (или временам) с пропагаторами $(23),(24)$ легко выполняется и дает совокупность квадратичных по импульсам множителей в знаменателе [6]. После этого возникает возможность перехода к представлению Фейнмана. Отличие от статического случая (модель (2)) состоит в том, что множители в знаменателе являются в данном случае суммой “энергий” $E_{k}$, и в то же время в пропагаторе $(24)$ множитель $E_{k}$ в знаменателе отсутствует. Мы рассмотрим на примере трехпетлевой диаграммы необходимые модификации для построения фейнмановского представления, а затем сформулируем общие правила его построения прямо по виду диаграммы.

В третьем порядке теории возмущений вклад в величину $f_{1}$ определяется единственной диаграммой

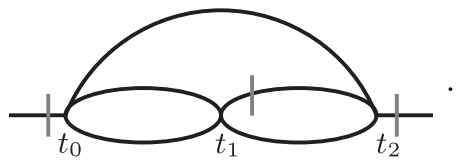

Диаграммы, определяющие вклад в $f_{1}$, вычисляются на нулевой частоте, для диаграммы (25) это соответствует взятию интеграла по всевозможным временам $t_{1}$ и $t_{2}$. С учетом $\Theta$-функции $\Theta\left(t_{2}-t_{1}\right)$ в линии $\psi \psi^{\prime}$ в диаграмме $(25)$ имеется три “временные версии": $t_{0} \leqslant t_{1} \leqslant t_{2}, t_{1} \leqslant t_{0} \leqslant t_{2}$ и $t_{1} \leqslant t_{2} \leqslant t_{0}$. Приведем графические изображения для этих временных версий, а также результат интегрирования по времени экспоненциальных множителей в пропагаторах (23), (24) в каждой из версий:

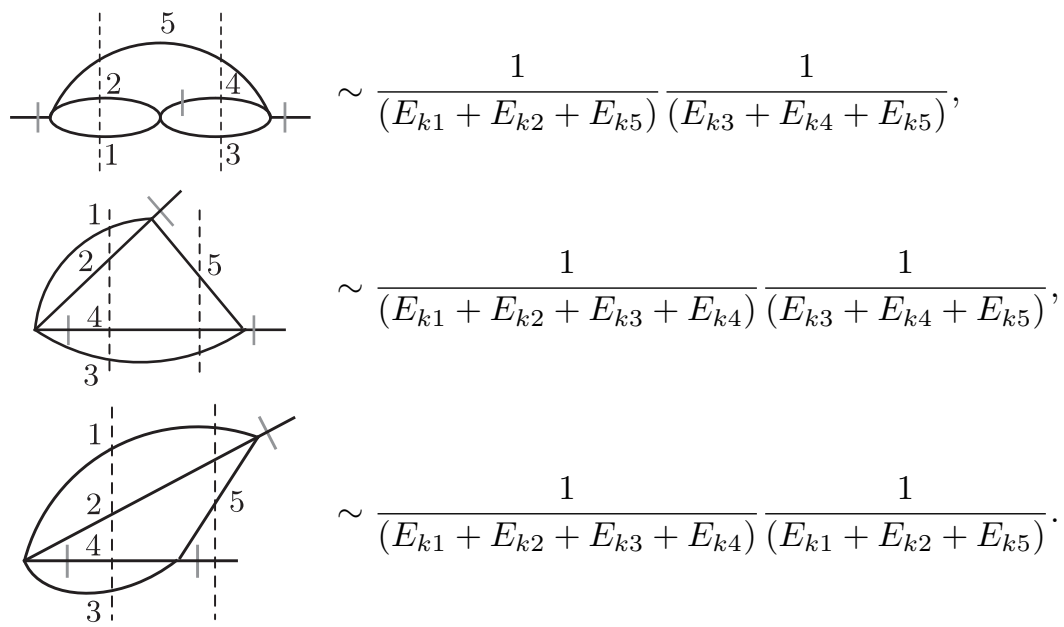

Проведенные между каждой парой соседних вершин вертикальные пунктиры ("сечения") определяют множители в знаменателях, равные сумме “энергий” $E_{k}$ линий в этом сечении [6].

Зависимость от импульсов интегрирования в диаграммах после проведения интегрирования по временам имеет структуру, позволяющую перейти к фейнмановскому представлению. Рассмотрим такой переход на примере временной версии (26). С учетом множителей $1 / E_{k}$ в пропагаторах (23) диаграмме сопоставляется интеграл

$$
J=\int d \mathbf{k}_{1} \cdots \int d \mathbf{k}_{5} \frac{\delta\left(\mathbf{k}_{1}+\mathbf{k}_{2}+\mathbf{k}_{5}\right) \delta\left(\mathbf{k}_{3}+\mathbf{k}_{4}+\mathbf{k}_{5}\right)}{E_{k_{1}} E_{k_{2}} E_{k_{3}} E_{k_{5}}\left(E_{k_{1}}+E_{k_{2}}+E_{k_{5}}\right)\left(E_{k_{3}}+E_{k_{4}}+E_{k_{5}}\right)} .
$$


Используя в выражении (29) формулу Фейнмана, получаем равенство

$$
J=\int_{0}^{1} \prod d v_{i} \delta\left(\sum_{i} v_{i}-1\right) F(\{v\})
$$

где

$$
\begin{aligned}
F(\{v\})= & \int d \mathbf{k}_{1} \cdots \int d \mathbf{k}_{5} \frac{\delta\left(\mathbf{k}_{1}+\mathbf{k}_{2}+\mathbf{k}_{5}\right) \delta\left(\mathbf{k}_{3}+\mathbf{k}_{4}+\mathbf{k}_{5}\right)}{Q^{\alpha}}, \\
Q= & v_{1} E_{k_{1}}+v_{2} E_{k_{2}}+v_{3} E_{k_{3}}+v_{5} E_{k_{5}}+ \\
& \quad+v_{6}\left(E_{k_{1}}+E_{k_{2}}+E_{k_{5}}\right)+v_{7}\left(E_{k_{3}}+E_{k_{4}}+E_{k_{5}}\right)
\end{aligned}
$$

$\alpha=6$ - число сомножителей в знаменателе формулы (29). Записывая $Q$ в виде

$$
Q=u_{1} E_{k_{1}}+u_{2} E_{k_{2}}+u_{3} E_{k_{3}}+u_{4} E_{k_{4}}+u_{5} E_{k_{5}}
$$

где

$$
\begin{gathered}
u_{1}=v_{1}+v_{6}, \quad u_{2}=v_{2}+v_{6}, \quad u_{3}=v_{3}+v_{7}, \\
u_{4}=v_{7}, \quad u_{5}=v_{5}+v_{6}+v_{7},
\end{gathered}
$$

из равенств (31), (33) получаем, что

$$
F(\{v\})=\int d \mathbf{k}_{1} \cdots d \mathbf{k}_{5} \frac{\delta\left(\mathbf{k}_{1}+\mathbf{k}_{2}+\mathbf{k}_{5}\right) \delta\left(\mathbf{k}_{3}+\mathbf{k}_{4}+\mathbf{k}_{5}\right)}{\left(u_{1} E_{k_{1}}+u_{2} E_{k_{2}}+u_{3} E_{k_{3}}+u_{4} E_{k_{4}}+u_{5} E_{k_{5}}\right)^{\alpha}} .
$$

Выбирая в формуле (35) в качестве независимых переменных некоторый набор $\left\{\mathbf{k}_{i_{1}}, \mathbf{k}_{i_{2}}, \mathbf{k}_{i_{3}}\right\}$ и выполняя интегрирования с помощью $\delta$-функций, приходим к выражению вида

$$
F(\{v\})=\int d \mathbf{k}_{i_{1}} \int d \mathbf{k}_{i_{2}} \int d \mathbf{k}_{i_{3}} \frac{1}{\left(C+V_{i_{j}, i_{l}} \mathbf{k}_{i_{j}} \mathbf{k}_{i_{l}}\right)^{\alpha}}, \quad C \equiv \tau \sum_{j=1}^{5} u_{j} .
$$

Вычисляя интеграл от степени квадратичной формы, получаем равенство

$$
F(\{v\})=\pi^{d n / 2} C^{3 d / 2-\alpha} \frac{\Gamma(\alpha-d n / 2)}{\Gamma(\alpha)}(\operatorname{det} V)^{-d / 2},
$$

где $n$ - число петель в диаграмме (в рассматриваемом случае $n=3$ ).

Значение детерминанта $\operatorname{det} V$ в выражении (37) не зависит от выбора переменных интегрирования $\left\{\mathbf{k}_{i_{1}}, \mathbf{k}_{i_{2}}, \mathbf{k}_{i_{3}}\right\}$, и его можно определить прямо по виду диаграммы. По построению $\operatorname{det} V$ представляет собой сумму произведений трех сомножителей $u_{i}$. Для любого набора независимых переменных интегрирования $\left\{\mathbf{k}_{i_{1}}, \mathbf{k}_{i_{2}}, \mathbf{k}_{i_{3}}\right\}$ диагональные элементы матрицы $V$ равны $u_{i_{1}}, u_{i_{2}}, u_{i_{3}}$, а их произведение дает вклад в $\operatorname{det} V$ с коэффициентом единица. Недиагональные элементы матрицы $V$ не содержат величин $u_{i_{1}}, u_{i_{2}}, u_{i_{3}}$, следовательно, $\operatorname{det} V$ не содержит старших степеней $u_{i}$. В качестве независимых переменных нельзя выбирать наборы импульсов, входящих в “законы сохранения" (аргументы $\delta$-функций в (35)). В рассматриваемом случае такими являются наборы $\left\{\mathbf{k}_{1}, \mathbf{k}_{2}, \mathbf{k}_{5}\right\},\left\{\mathbf{k}_{3}, \mathbf{k}_{4}, \mathbf{k}_{5}\right\}$, и соответствующие произведения $u_{1} u_{2} u_{5}$ и $u_{3} u_{4} u_{5}$ отсутствуют в $\operatorname{det} V$. Таким образом, для диаграммы $(25) \operatorname{det} V$ дается выражением

$\operatorname{det} V=u_{1} u_{2} u_{3}+u_{1} u_{2} u_{4}+u_{1} u_{3} u_{4}+u_{1} u_{3} u_{5}+u_{1} u_{4} u_{5}+u_{2} u_{3} u_{4}+u_{2} u_{3} u_{5}+u_{2} u_{4} u_{5}$, 
в котором величины $u_{i}$ необходимо выразить через $v_{i}$ согласно формуле (34). Во временных версиях (27) и (28) выражение (38) сохраняется, изменяются соответствующим образом лишь связи переменных $u_{i}$ и $v_{i}$, которые легко можно найти по виду диаграмм.

Заметим, что выражения (33), (38) совпадают по виду с соответствующими выражениями для статической диаграммы (25) (без перечеркнутых линий) теории $\phi^{4}$.

\section{R-ОПЕРАЦИЯ ПОСЛЕ ИНТЕГРИРОВАНИЯ ПО ВРЕМЕНИ}

В принятой схеме ренормировки вычитания из расходящихся подграфов проводятся на нулевой частоте, втекающей в подграф. В формулировке $(22)$ это включает в себя процедуру растяжения втекающей в подграф частоты $\omega \rightarrow a \omega$. Чтобы интерпретировать такое растяжение в импульсно-временном представлении и выполнить интегрирование по временам с использованием временных версий, это растяжение можно заменить соответствующим изменением подынтегрального выражения подграфа. Рассмотрим в качестве примера четыреххвостый подграф, образованный линиями 3 и 4, во временной версии (26). Зависимость подграфа от втекающей частоты $\omega$ определяется интегралом

$$
\int_{0}^{\infty} d t \exp \left[-t\left(i a \omega+\lambda E_{k_{3}}+\lambda E_{k_{4}}\right)\right]=\frac{1}{a} \int_{0}^{\infty} d t \exp \left[-t\left(i \omega+\lambda \frac{E_{k_{3}}}{a}+\lambda \frac{E_{k_{4}}}{a}\right)\right],
$$

где $a$ - параметр растяжения, $t=t_{1}-t_{2}$ (см. формулу (25)) и учтена зависимость от времени пропагаторов (23), (24). Правая часть равенства, полученная заменой $t a \rightarrow t$, показывает, что растяжение втекающей в подграф частоты эквивалентно замене $E_{k} \rightarrow E_{k} / a$ во всех экспонециальных множителях пропагаторов линий $(23),(24)$, входящих в подграф, а также деления на $a$ всего подынтегрального выражения. После такого преобразования подынтегрального выражения для подграфа диаграмму можно вычислить в импульсно-временном представлении. Вклад правого сечения во временной версии (26) заменяется на

$$
\frac{1}{a} \frac{1}{E_{k_{3}} / a+E_{k_{4}} / a+E_{k_{5}}}=\frac{1}{E_{k_{3}}+E_{k_{4}}+a E_{k_{5}}} .
$$

Действуя аналогичным образом для временной версии (27), находим, что меняются оба сомножителя в знаменателе, что дает равенство

$$
\begin{aligned}
& \frac{1}{a} \frac{1}{\left(E_{k_{3}} / a+E_{k_{4}} / a+E_{k_{1}}+E_{k_{2}}\right)\left(E_{k_{3}} / a+E_{k_{4}} / a+E_{k_{5}}\right)}= \\
& =\frac{a}{\left(E_{k_{3}}+E_{k_{4}}+a E_{k_{1}}+a E_{k_{2}}\right)\left(E_{k_{3}}+E_{k_{4}}+a E_{k_{5}}\right)} \text {. }
\end{aligned}
$$

Учитывая, что рассматриваемый подграф является логарифмическим, действие операции (22) на (40) приводит к выражению

$$
\begin{aligned}
\int_{0}^{1} d a \partial_{a}\left(\frac{a}{\left(E_{k_{3}}+E_{k_{4}}+a E_{k_{1}}+a E_{k_{2}}\right)\left(E_{k_{3}}+E_{k_{4}}+a E_{k_{5}}\right)}\right)= \\
=\frac{1}{\left(E_{k_{3}}+E_{k_{4}}+E_{k_{1}}+E_{k_{2}}\right) E_{k_{3}}+E_{k_{4}}+E_{k_{5}}} .
\end{aligned}
$$

Это означает, что контрчлен к этой временной версии не нужен. 
Рассмотренные примеры легко обобщаются на многопетлевые подграфы. Учет растяжения в $a$ раз втекающей в подграф частоты в терминах интегрирования по временным версиям заменяется на введение множителя $a$ перед всеми входящими в сечения энергиями $E_{k_{j}}$, не принадлежащими подграфу. Если число сечений, проходящих через подграф, превышает число сечений уединенного подграфа, то на каждое дополнительное сечение вводится дополнительный множитель $a$. Для логарифмических подграфов соответствующая временная версия не требует в этом случае введения растяжения. Для квадратично расходящихся подграфов одно дополнительное сечение делает данную временную версию логарифмической и понижает до $n_{i}=0$ значение параметра в операции (22). В случае двух и более дополнительных сечений контрчлен к данной временной версии не нужен.

Для окончательного представления $R^{\prime}$-операции в форме $(22)$ в импульсном представлении после интегрирования по временным версиям необходимо также провести растяжения втекающих в расходящиеся подграфы импульсов. В фейнмановском представлении этому соответствует следующее правило расстановки множителей $a$ в слагаемых статического детерминанта (38) [8]. Для $n^{\prime}$-петлевого подграфа $n$-петлевой диаграммы множитель $a^{N-n^{\prime}}$ ставится перед слагаемыми детерминанта, содержащими $N>n^{\prime}$ параметров $u_{i}$, принадлежащих рассматриваемому подграфу. Для диаграммы (26) это дает равенство

$$
\operatorname{det} V_{a}=u_{1} u_{2} u_{3}+u_{1} u_{2} u_{4}+a u_{1} u_{3} u_{4}+u_{1} u_{3} u_{5}+u_{1} u_{4} u_{5}+a u_{2} u_{3} u_{4}+u_{2} u_{3} u_{5}+u_{2} u_{4} u_{5} .
$$

Окончательный вид детерминанта с растяжением для временной версии (26) можно получить, выражая в формуле (42) параметры $u_{i}$ через $v_{j}$ с помощью соотношения (34), в которое введено растяжение $a$ в соответствие с изменением сечения (39):

$$
u_{1}=v_{1}+v_{6}, \quad u_{2}=v_{2}+v_{6}, \quad u_{3}=v_{3}+v_{7}, \quad u_{4}=v_{7}, \quad u_{5}=v_{5}+v_{6}+a v_{7} .
$$

В итоге в терминах параметров $v_{i}$ получаем, что

$$
\begin{aligned}
\operatorname{det} V_{a}= & v_{1} v_{2} v_{3}+2 v_{1} v_{2} v_{7}+v_{1} v_{3} v_{5}+2 v_{1} v_{3} v_{6}+2 a v_{1} v_{3} v_{7}+2 v_{1} v_{5} v_{7}+ \\
& +4 v_{1} v_{6} v_{7}+3 a v_{1} v_{7}^{2}+v_{2} v_{3} v_{5}+2 v_{2} v_{3} v_{6}+2 a v_{2} v_{3} v_{7}+2 v_{2} v_{5} v_{7}+ \\
& +4 v_{2} v_{6} v_{7}+3 a v_{2} v_{7}^{2}+2 v_{3} v_{5} v_{6}+3 v_{3} v_{6}^{2}+ \\
& +4 a v_{3} v_{6} v_{7}+4 v_{5} v_{6} v_{7}+6 v_{6}^{2} v_{7}+6 a v_{6} v_{7}^{2} .
\end{aligned}
$$

\section{5. РЕЗУЛЬТАТЫ}

Результаты расчетов по теории возмущений мы приводим в терминах заряда $u=$ $\left(S_{d} /(2 \pi)^{d}\right) g$, где $S_{d}=2 \pi^{d / 2} / \Gamma(d / 2)$ - площадь $d$-мерной сферы единичного радиуса. Динамический критический индекс $z$ определяется выражением

$$
z=2-\gamma_{\lambda}^{*}
$$

где $\gamma_{\lambda}^{*} \equiv \gamma_{\lambda}\left(u_{*}\right)$ - значение РГ-функции $\gamma_{\lambda}(u)$ в неподвижной точке $u=u_{*}$ (корне уравнения $\left.\beta\left(u_{*}\right)=0\right)$. Используя формулу (13) и учитывая, что величина $2 \gamma_{\psi}^{*}=\eta$ определяет известный статический индекс Фишера $\eta$, имеем

$$
\gamma_{\lambda}^{*}=\eta-\gamma_{1}^{*}, \quad \eta=\gamma_{3}^{*}
$$


Используя для нахождения РГ-функций соотношения (16) находим, что

$$
\gamma_{1}^{*}=\frac{2 f_{1}^{*}}{1+f_{3}^{*}}, \quad \gamma_{3}^{*}=\frac{2 f_{3}^{*}}{1+f_{3}^{*}} .
$$

Выражая $f_{3}^{*}$ через $\eta$ с использованием выражений (46), (47), получаем окончательно, что

$$
\gamma_{\lambda}^{*}=\eta-(2-\eta) f_{1}^{*} .
$$

В рассматриваемой схеме ренормировки значение заряда в неподвижной точке $u_{*}$ рассчитано численно для $n$-компонентного поля $\psi$ с пятипетлевой точностью в работе [9]. Необходимые для наших целей три члена $\varepsilon$-разложения величины $u_{*}$ даются аналитическим выражением

$$
\begin{aligned}
u_{*}= & \frac{2 \varepsilon}{3 k_{1}}+\frac{\varepsilon^{2}}{81 k_{1}^{3}}\left(27 k_{1}^{2}-2 k_{3}+36 k_{4}\right)+\frac{\varepsilon^{3}}{27 k_{1}^{5}}\left[\frac{41}{48} k_{1}^{2} k_{3}+\frac{4}{81} k_{3}^{2}+16 k_{4}^{2}-\right. \\
& -\frac{16}{9} k_{3} k_{4}-\frac{5015}{1728} k_{1}^{4}-8 \zeta(3) k_{1} k_{4}+\frac{75}{32} k_{1} k_{5}- \\
& \left.-\frac{181}{16} k_{1} k_{6}-\left(\frac{25}{96}+\frac{3}{2} \zeta(3)\right) k_{1}^{2} k_{4}\right]
\end{aligned}
$$

где

$$
\begin{gathered}
k_{1}=\frac{n+8}{9}, \quad k_{2}=\frac{n^{2}+6 n+20}{27}, \quad k_{3}=\frac{n+2}{3}, \quad k_{4}=\frac{5 n+22}{27}, \\
k_{5}=\frac{3 n^{2}+22 n+56}{81}, \quad k_{6}=\frac{n^{2}+20 n+60}{81} .
\end{gathered}
$$

Индекс Фишера в четвертом порядке $\varepsilon$-разложения определяется выражением

$$
\begin{aligned}
\eta=\frac{\varepsilon^{2}(n+2)}{2(n+8)^{2}}(1 & +\frac{\varepsilon}{4(n+8)^{2}}\left(-n^{2}+56 n+272\right)+\frac{\varepsilon^{2}}{16(n+8)^{4}}\left(-5 n^{4}-230 n^{3}+\right. \\
& \left.\left.+1124 n^{2}+17920 n+46144-384(n+8)(5 n+22) \zeta(3)\right)\right) .
\end{aligned}
$$

Результаты расчета величины $f_{1}(u)$ в четвертом порядке теории возмущений представлены в табл. $1^{1)}$ :

$$
\begin{aligned}
2 f_{1}(u)= & \left(a_{1}+a_{2} \varepsilon+a_{3} \varepsilon^{2}\right) k_{3} u^{2}-\left(a_{4}+a_{5} \varepsilon\right) k_{1} k_{3} u^{3}+ \\
& +\left(a_{6} k_{2} k_{3}+a_{7} k_{3}^{2}+a_{8} k_{3} k_{4}+a_{9} k_{3} k_{4}\right) u^{4} .
\end{aligned}
$$

Двух- и трехпетлевое приближения представлены единственными диаграммами 1 и 2 соответственно. Каждой из четырехпетлевых диаграмм соответствует сумма вкладов со всевозможными перечеркиваниями одной из линий в каждой из внутренних вершин. Функции $f(n)$ определяют дополнительные множители при каждой из диаграмм, отличающие $n$-компонентный случай от однокомпонентного. Эти множители определяются только топологией диаграмм и совпадают с соответствующими множителями статической модели (все $k_{i}=1$ при $\left.n=1\right)$.

\footnotetext{
1) Детали расчета приведены в приложении.
} 
ТАБЛИЦА 1

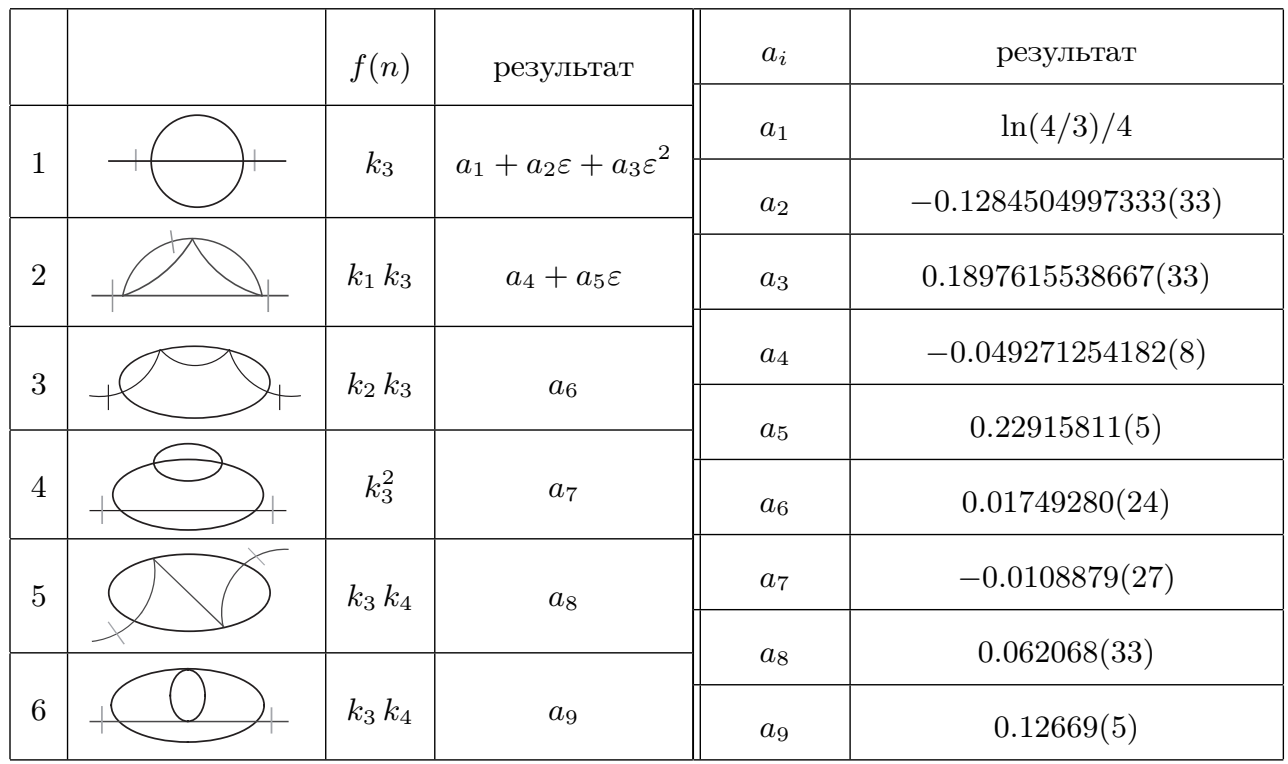

Результаты расчета динамического индекса, как правило, представляют в виде $z=2+R \eta$. Учитывая соотношения (45), (48), для величины $R$ получаем равенство

$$
R=\frac{2 f_{1}^{*}(1-\eta / 2)}{\eta-1} .
$$

Подставляя в это выражение формулы (49), (51), (52), находим три члена $\varepsilon$-разложения величины $R$ :

$$
R=\left(6 \ln \frac{4}{3}-1\right)\left(1+c_{1} \varepsilon+c_{2} \varepsilon^{2}+\frac{\varepsilon^{2}\left(c_{3}+c_{4} n\right)}{(n+8)^{2}}\right),
$$

где коэффициенты $c_{i} \equiv C_{i} /[6 \ln (4 / 3)-1]$ определяются соотношениями

$$
\begin{aligned}
& C_{1}=30 a_{1}+24 a_{2}-16 a_{4}, \\
& C_{2}=\frac{8593}{648} a_{1}+30 a_{2}+24 a_{3}-28 a_{4}-16 a_{5}+32 a_{6}, \\
& C_{3}=-\left(\frac{4030}{3}+264 \zeta(3)\right) a_{1}-672 a_{4}-1408 a_{6}+576 a_{7}+704 a_{8}+704 a_{9}, \\
& C_{4}=-\left(\frac{830}{3}+60 \zeta(3)\right) a_{1}-144 a_{4}-320 a_{6}+288 a_{7}+160 a_{8}+160 a_{9} .
\end{aligned}
$$

Первые два члена $\varepsilon$-разложения (54) не зависят от числа компонент поля $n$. Первый из них был вычислен в работе [10], второй - в статье [11]. В работе [10] величина $R$ вычислена также в главном порядке $1 / n$-разложения для произвольной размерности $d$ :

$$
R_{\infty}=\frac{4}{4-d}\left(\frac{d \Gamma^{2}(d / 2-1) / \Gamma(d-2)}{8 \int_{0}^{1 / 2} d x[x(2-x)]^{d / 2-2}}-1\right) .
$$


Первые члены разложения этой величины по $\varepsilon=4-d$ имеют вид

$$
R_{\infty}=\left(6 \ln \frac{4}{3}-1\right)\left(1-0.188483417 \varepsilon-0.099952926 \varepsilon^{2}+O\left(\varepsilon^{3}\right)\right)
$$

С учетом того, что первые два члена $\varepsilon$-разложения (54) не зависят от $n$, они совпадают с соответствующими вкладами в формуле (60), что подтверждается результатами работ [10], [11]. Соотношение (60) определяет также коэффициент $c_{2}$ в квадратичном по $\varepsilon$ вкладе из равенства (54), следовательно,

$$
c_{1}=-0.188483417, \quad c_{2}=-0.099952926 .
$$

Для проверки точности расчетов диаграмм в табл. 1 мы рассчитали коэффициенты $c_{1}$, и $c_{2}$, используя соотношения (55), (56). Подставляя данные из таблицы, находим значения $c_{1}=-0.18848341720(21), c_{2}=-0.100096(11)$, что с хорошей точностью совпадает со значениями (61). Для коэффициентов $c_{3}, c_{4}(57),(58)$ получаем равенства

$$
c_{3}=21.56(6), \quad c_{4}=4.788(13) .
$$

Впервые эти коэффициенты были рассчитаны в работе [12]. Вычисления в этой работе проводились в импульсном представлении в схеме $M S$, точность расчета составила $1 \div 2 \%$. В рамках погрешности полученные нами значения (62) согласуются с результатами работы [12], но имеют значительно меньшую погрешность.

Приведем для полноты картины полученное $\varepsilon$-разложение динамического индекса $z$ для случая $n=1$ :

$$
z=2+0.013446156198(1) \varepsilon^{2}+0.0110362801761(28) \varepsilon^{3}-0.005576(12) \varepsilon^{4} .
$$

\section{6. ЗАКЛЮЧЕНИЕ}

В настоящей работе техника вычисления РГ-функций путем представления диаграмм Фейнмана в виде несингулярных интегралов использована для выполнения четырехпетлевого расчета модели А критической динамики. Вычисление четырехпетлевых диаграмм проводилось численно с помощью техники Sector Decomposition, обобщенной на задачи критической динамики. С этой целью был получен рецепт записи диаграмм (с учетом контрчленов) в фейнмановском представлении непосредственно по виду диаграмм. Использованная техника позволила впервые произвести четырехпетлевый расчет в модели критической динамики с точностью порядка $0.3 \%$.

\section{ПРИЛОЖЕНИЕ}

Использование формулы (22) для $R$-операции при вычислении величины $f_{1}$ по диаграммам 1-6 в табл. 1 позволяет получить их представление в виде интегралов, не содержащих расходимостей по $\varepsilon$, как в импульсном, так и фейнмановском представлении. Оказалось, что диаграммы 1-3, не содержащие вложенных подграфов, более выгодно вычислять в импульсном представлении, а затем можно выполнить в них явное интегрирование по параметрам $a$. Используя симметричное протекание втекающего в однопетлевые подграфы импульса, удалось исключить скалярные произведения из выражений для сечений временных версий и выполнить аналитическое интегрирование по оставшимся углам при $\varepsilon=0$, после чего осталось лишь 
численно рассчитать четырехкратный интеграл по модулям импульсов. В данном случае вычитание контрчленов в подынтегральном выражении носит простой характер и не препятствует численному расчету. При желании можно легко провести явное сокращение плохо убывающих вкладов в подынтегральном выражении.

В диаграммах 4-6 в табл. 1 такое сокращение произвести затруднительно, и было осуществлено произведено дифференцирование по параметрам $a$ подынтегральных функций, что заведомо дает выражение, свободное от расходимостей. Вычисления этих диаграмм проводились в фейнмановском представлении техникой Sector Decomposition. В качестве стратегии использовались секторы Спира [13], которые для динамических диаграмм с полиномами Симанчика типа (44) можно легко получить из секторов статических диаграмм благодаря наличию замен (43), связывающих полиномы (42) и (44). Полученные "динамические" секторы следует дополнить последующими шагами декомпозиции из-за наличия в полиномах параметров растяжения $a$. Построение данных шагов декомпозиции подробно рассмотрено в работе [8].

Благодарности. Исследования были проведены с использованием вычислительных ресурсов Ресурсного центра "Вычислительный центр СПбГУ".

\section{Список литературы}

[1] Л.Ц. Аджемян, М.В. Компаниец, "Ренормгруппа и $\varepsilon$-разложение: представление $\beta$-функции и аномальных размерностей несингулярными интегралами”, ТМФ, 169:1 (2011), 100-111.

[2] Л.Ц. Аджемян, М. В. Компаниец, С. В. Новиков, В. К. Сазонов, "Представление $\beta$-функции и аномальных размерностей несингулярными интегралами: доказательство основного соотношения", ТМФ, 175:3 (2013), 325-336.

[3] Л. Ц. Аджемян, С. Е. Воробьева, М. В. Компаниец, "Представление несингулярными интегралами $\beta$-функции и аномальных размерностей в моделях критической динамики", ТМФ, 185:1 (2015), 3-11.

[4] P. C. Hohenberg, B. I. Halperin, "Theory of dynamic critical phenomena", Rev. Modern Phys., 49:3 (1977), 435-479.

[5] T. Binoth, G. Heinrich, "An automatized algorithm to compute infrared divergent multi-loop integrals", Nucl. Phys. B, 585:3 (2000), 741-759.

[6] А.Н. Васильев, Квантовополевая ренормгруппа в теории критического поведения и стохастической динамике, ПИЯФ, СПб., 1998.

[7] О. И. Завьялов, Перенормированные диаграммы Фейнмана, Наука, М., 1979.

[8] L. Ts. Adzhemyan, Yu. V. Kirienko, M. V. Kompaniets, "Critical exponent $\eta$ in 2D $O(N)$-symmetric $\varphi^{4}$-model up to 6 loops", arXiv: 1602.02324 .

[9] L. Ts. Adzhemyan, M. V. Kompaniets, "Five-loop numerical evaluation of critical exponents of the $\varphi^{4}$ theory", J. Phys.: Conf. Ser., 523:1 (2014), 012049, 9 pp.

[10] B. I. Halperin, P. C. Hohenberg, S.-K. Ma, "Calculation of dynamic critical properties using Wilson's expansion methods", Phys. Rev. Lett., 29:23 (1972), 1548-1551.

[11] Н. В. Антонов, А. Н. Васильев, "Критическая динамика как теория поля", ТМФ, 60:1 (1984), 59-71.

[12] Л. Ц. Аджемян, С. В. Новиков, Л. Сладкофф, "Расчет динамического индекса модели $A$ критической динамики в порядке $\varepsilon^{4} "$, Вестн. С.-Петербург. ун-та. Сер. 4. Физика, Химия, 2008, № 4, 109-112.

[13] E. R. Speer, "Mass singularities of generic Feynman amplitudes", Ann. Inst. Henri Poincaré Sect. A (N.S.), 26:1 (1977), 87-105. 